\title{
Jahresbericht der AOTrauma Deutschland
}

\author{
Michael J. Raschke, Philip Wilbrandt
}

Die AOTrauma Deutschland hat ihre Aktivitäten und Mitgliedschaft weiter ausgebaut. Die Nachwuchsförderung wurde intensiviert. Die Akademie wird in 2016 erstmals eigene Veranstaltungen anbieten.

\section{Jahrestagung 2016}

Vom 5. bis 7. Mai 2016 fand in Bern, Schweiz, die Jahrestagung mit 136 Mitgliedern statt. Es war das alle 4 Jahre stattfindende 3-Länder-Treffen mit der AOTrauma Schweiz und Österreich.

Jedes Land gestaltete einen halben Tag das wissenschaftliche Programm. Die anhand von vielen Fällen diskutierten Themen waren proximaler Humerus (Österreich), Hüfte (Schweiz) und Ellbogen (Deutschland). Für den deutschen Programmteil wurden über eine öffentliche Ausschreibung 3 Reisestipendien zur Jahrestagung vergeben. Diese erhielten: Anna Schreiner (Tübingen), Martin Jordan (Würzburg) und Johannes Habering (BGU Frankfurt).

\section{Gemeinsame Projekte der D-A-CH-Länder}

Abgesehen von der 3-Länder-Tagung veranstalten die D-A-CH-Länder gemeinsam jedes Jahr im Dezember den deutschsprachigen AOTrauma-Masterkurs in Davos und geben das OP-Journal heraus.

In Bern wurde entschieden, die gemeinsame Jahrestagung öfter abzuhalten. Geplant ist, sie ab 2019 alle 2 Jahre stattfinden zu lassen. Weiterhin wurde eine europäische AOTrauma-Tagung angeregt.

Ab 2017 wird - zusätzlich zum deutschsprachigen AOTrauma-Masterkurs in Davos - ein weiterer in Deutschland/Öster-

OP-JOURNAL 2016; 32: 65-69

(C) Georg Thieme Verlag KG Stuttgart · New York DOI http://dx.doi.org/10.1055/s-0042-108376

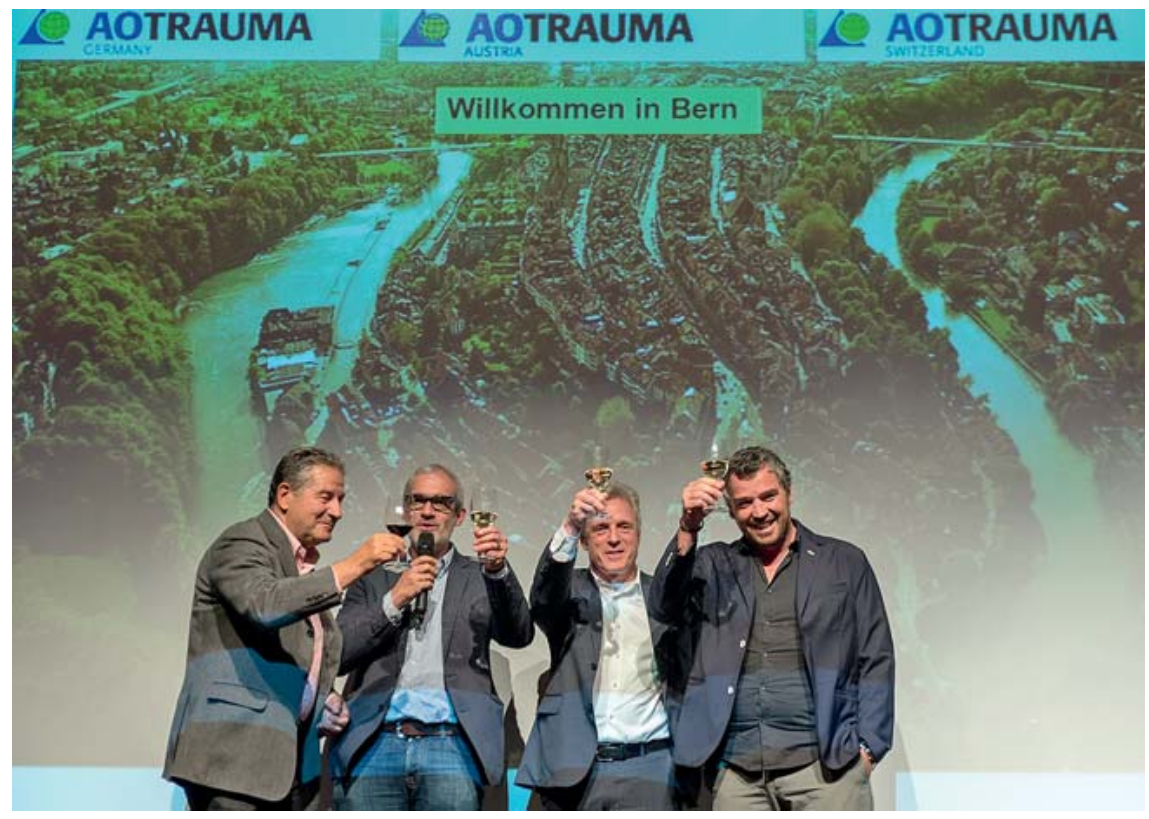

Abb. 1 Wagner (Präsident A), Keel (Gastgeber), Raschke (Präsident D) und Borens (Präsident $\mathrm{CH})$ (Foto: Jürgen Staiger).

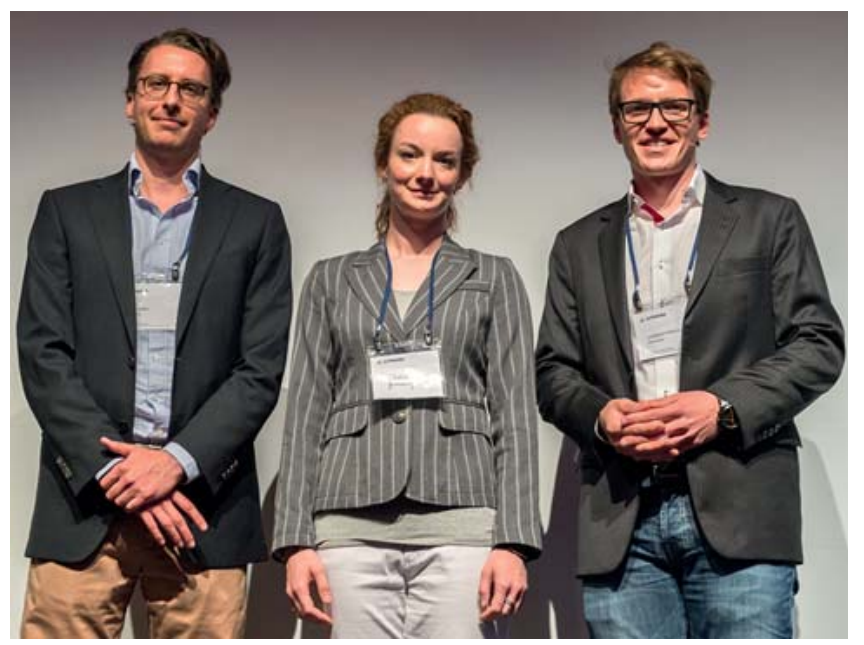

Abb. 2 Jordan, Schreiner und Habering (Foto: Jürgen Staiger). reich angeboten. Erstmals wird dieser vom 12. bis 14. Juni 2017 in Dresden stattfinden. Die deutschen Kursleiter sind Felix Bonnaire und Klaus Schaser aus Dresden, aus der Schweiz Andreas Platz sowie aus Österreich Franz Kralinger bzw. Franz Seibert.
Eine knappe Mehrheit der Teilnehmer sah keinen Bedarf für eine Neuauflage des vergriffenen deutschsprachigen AO Manuals bzw. eine deutschsprachige Ausgabe der kommenden, überarbeiteten Neuauflage. 


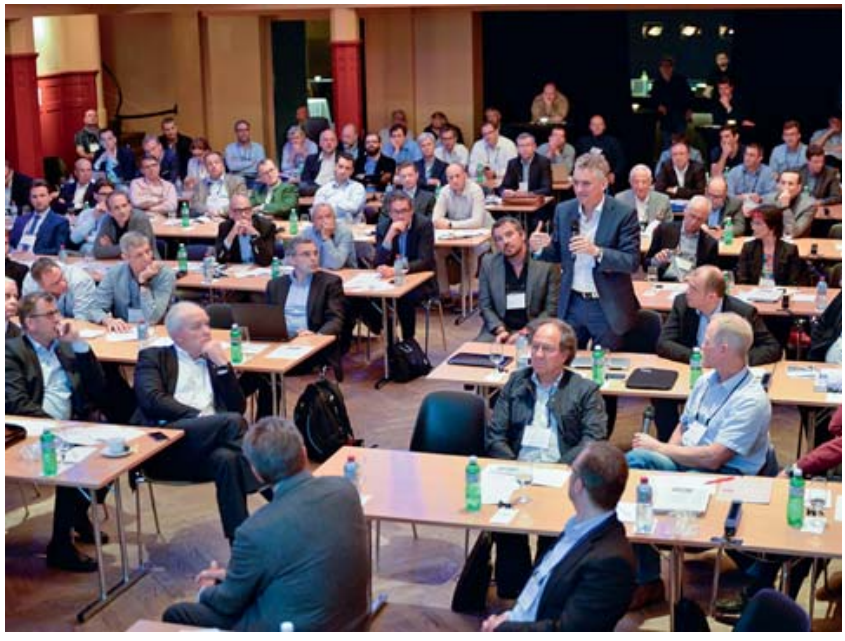

Abb. 3 Diskussion über gemeinsame Projekte (Foto: Jürgen Staiger).

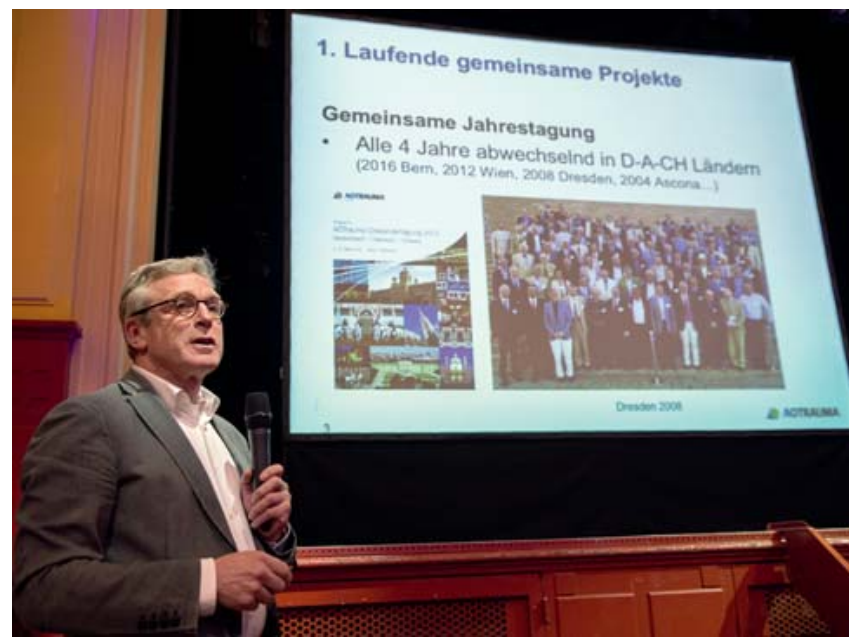

Abb. 4 Raschke präsentiert Vorschläge für neue gemeinsame Aktivitäten (Foto: Jürgen Staiger).
Die Vorstände der D-A-CH-Länder erhielten einstimmig das Mandat, bei den verantwortlichen Personen und Gremien von AOTrauma auf eine Veränderung der Stimmverhältnisse innerhalb Europas einzuwirken, da diese bisher nicht die Anzahl der Kurse, FellowshipZentren und sonstigen Aktivitäten der $\mathrm{D}-\mathrm{A}-\mathrm{CH}$ und anderer, besonders aktiver Länder angemessen berücksichtigen.
Am Ende der D-A-CH-Sitzung erhielt jeder Teilnehmer ein Exemplar des Buches von Eugen Kuner „Vom Ende einer qualvollen Therapie im Streckverband“, das von der AOTrauma Deutschland mit einem Druckkostenzuschuss gefördert wurde.

Während des Festabends auf dem Gurten wurde Lothar Kinzl (Ulm) zum Ehrenmitglied der AOTrauma Deutschland ernannt.

\section{Präsidium und Beirat}

Es standen keine Wahlen an. Die Amtszeit des für 3 Jahre gewählten Geschäftsführenden Präsidiums dauert noch bis Ende 2018. Es setzt sich zusammen aus: Michael Raschke (Präsident, Münster), Hans-Jörg Oestern (Past-Präsident, Celle), Florian Gebhard (Vize-Präsident, Ulm) und Ulrich Stöckle (Schatzmeister, Tübingen).

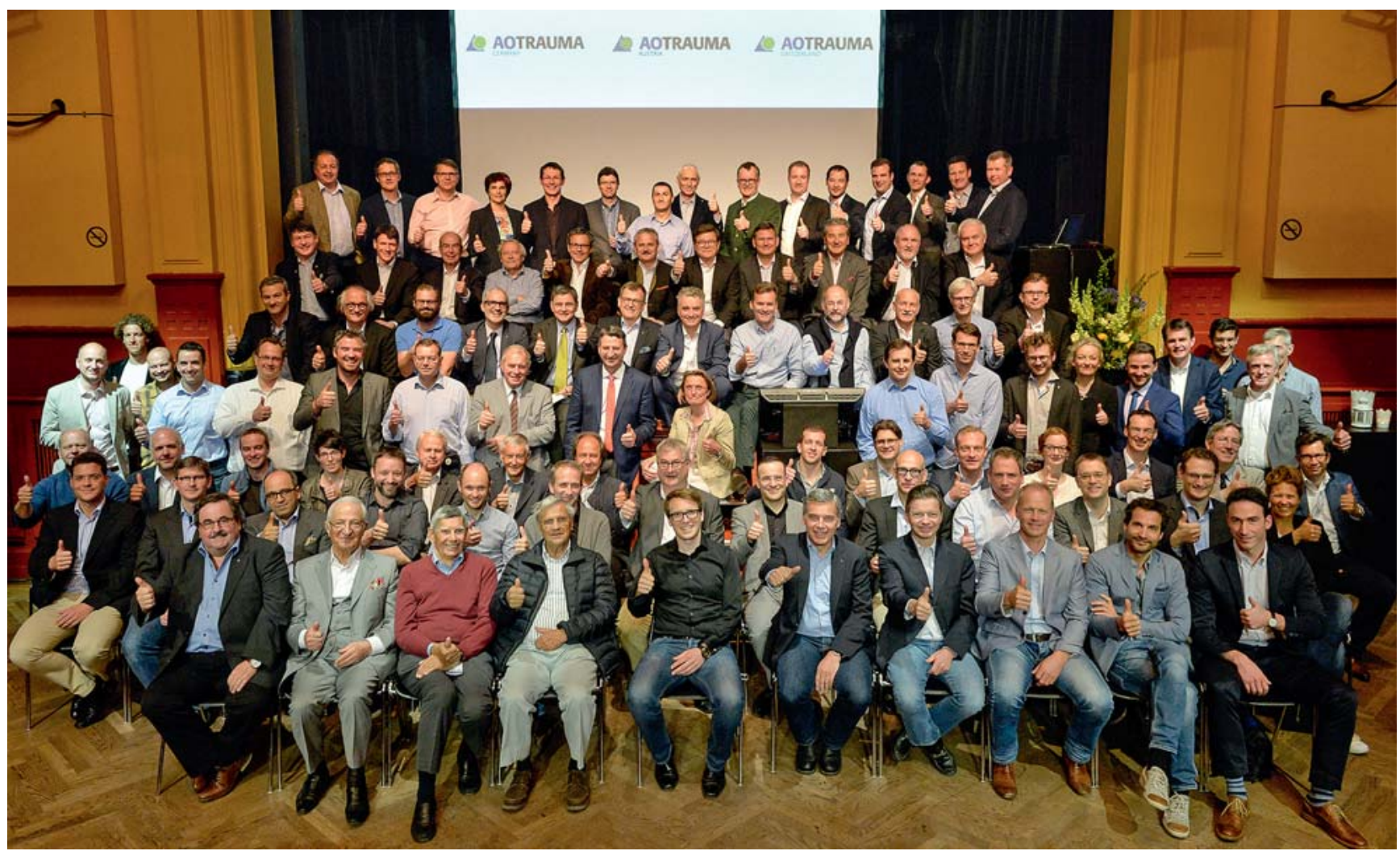

Abb.5 Teilnehmer der 3-Länder-Tagung (Foto: Jürgen Staiger). 


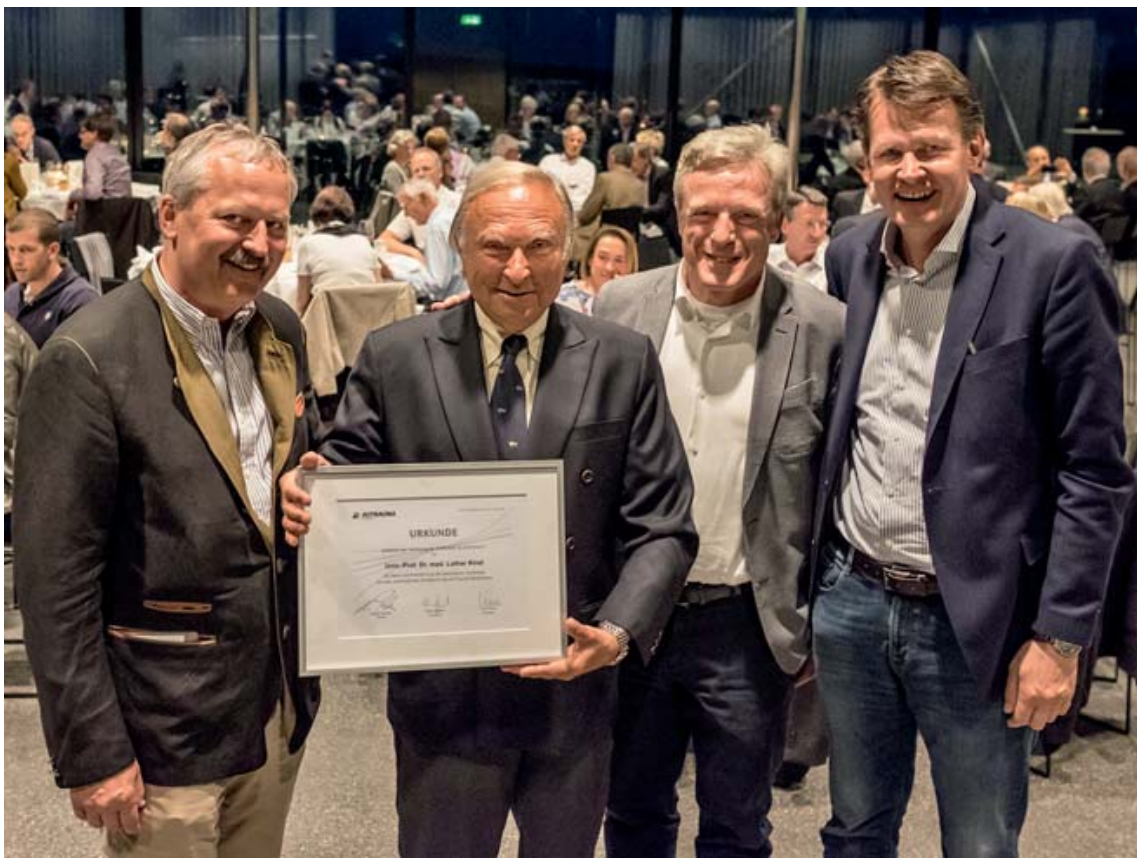

Abb. 6 Gebhard, Kinzl, Raschke und Stöckle (Foto: Jürgen Staiger).

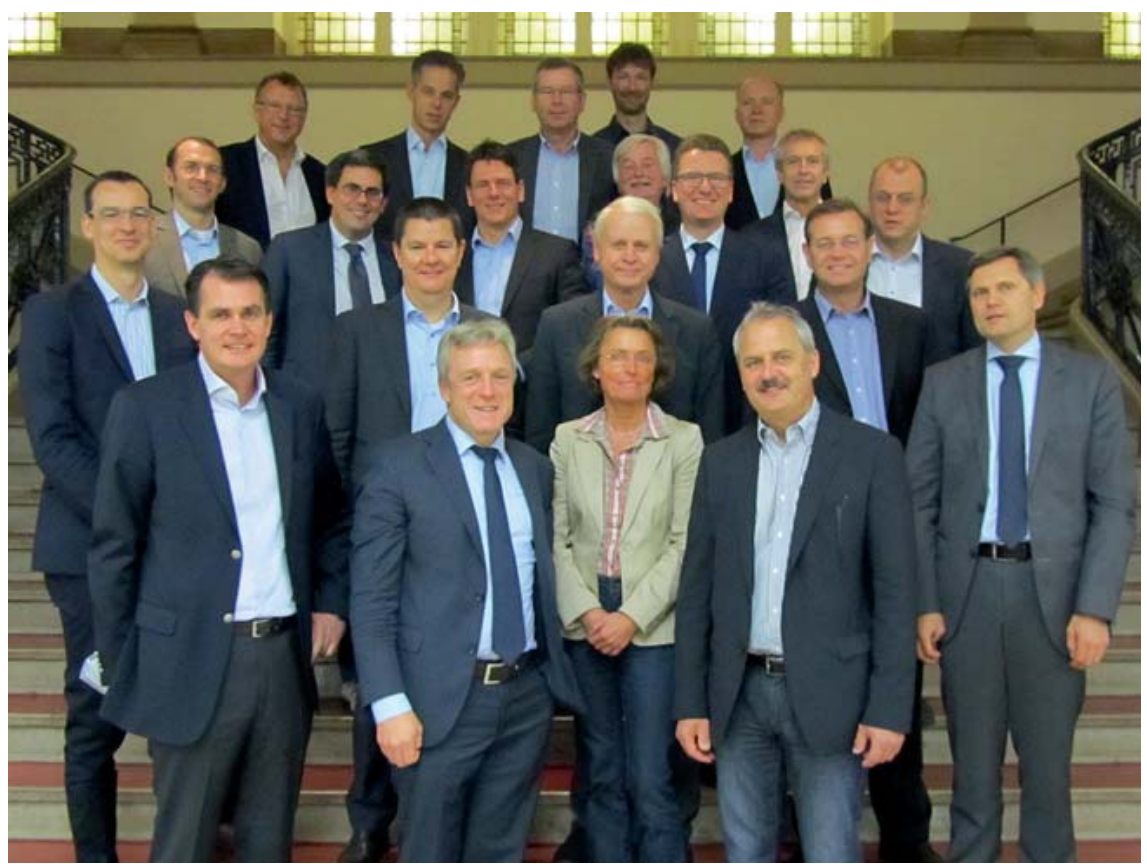

Abb. 7 Präsidium und Beirat (Foto: Philip Wilbrandt).

Zum Erweiterten Präsidium gehören zusätzlich: Georg Duda, Berlin (Forschung, Berlin), Karl-Heinz Frosch (Community Development, Hamburg), Heiner Winker (Senior Education Officer, Erfurt), Markus Schultheiss (Spine, Ulm), Max Heiland, Hamburg (CMF, Hamburg) und Andrea Meyer-Lindenberg (VET, München).

Der Beirat besteht aus 12 Personen: Felix Bonnaire (Dresden), Ulf Culemann (Celle), Bernd Füchtmeier (Regensburg),
AOTrauma Deutschland aufgenommen: Christian Kleber (Dresden), Peter Laier (Karlsruhe), Martin Langer (Münster), Tim Lögters (Düsseldorf), Wolfgang Schneiders (Dresden), Michael Schütz (Berlin), Philipp Schwabe (Berlin), Tim Schlummer (Warendorf) und Mohammad Tezval (Recklinghausen).

\section{Akademie: neue Aufnahmekriterien}

Eine Task Force hat die Aufnahmekriterien für die Akademie überarbeitet. Diese sind:

Für Ärzte

- Leiter einer „AO-Klinik“

- ansonsten (alle Kriterien müssen erfüllt sein):

- Facharzt für Orthopädie und Unfallchirurgie, Zusatzbezeichnung „spezielle Unfallchirurgie“

- Leitender Arzt/Chefarzt, aktuelle klinische Tätigkeit in einem zertifizierten Traumazentrum

- aktives Mitglied in der AOT Faculty und/oder einem AO-Gremium

- wissenschaftliche Publikationen

Für Wissenschaftler (alle Kriterien müssen erfüllt sein):

- leitende Position einer ausgewiesenen Forschungseinrichtung

- Forschungsleistung auf dem Gebiet der muskuloskeletalen Chirurgie/Osteologie

\section{Akademie: zukünftige Aktivitäten}

- Symposium auf dem DKOU 2016 „Wohin entwickelt sich die Unfallchirurgie?"

- Exklusive Teilnahme nur für Akademiemitglieder am AO-Expertensymposium in Tübingen am 7./8. Oktober 2016 in Tübingen.

- Ab 2017 Treffen vor der Jahrestagung (Nachmittag und Abendessen am Tag vor der Jahrestagung und Vormittag bis Start der Jahrestagung).

\section{Fortbildung: Kurse und Seminare und Faculty-Weiterqualifizierung}

Thomas Gösling (Braunschweig), Freddy Grützner (Ludwigshafen), Wolfgang Linhart (Heilbronn), Edgar Mayr (Augsburg), Thomas Mückley (Erfurt), Lars Peter Müller (Köln), Fabian Stuby (Tübingen), Klaus-Dieter Schaser (Dresden) und Joachim Windolf (Düsseldorf).

\section{Akademie: neue Mitglieder}

Auf der Jahrestagung wurden die folgenden 9 Mitglieder in die Akademie der
Im Jahr 2015 wurden 35 Veranstaltungen (Kurse und Seminare) an 81 Tagen organisiert. Diese wurden von 2076 Kursteilnehmern besucht. Insgesamt 553 unterschiedliche Personen (367 Referenten und 186 Instruktoren) trugen mit insgesamt 852 Einsätzen zum Erfolg der Kurse und Seminare bei. Dies bedeutet eine Steigerung in allen Bereichen. 


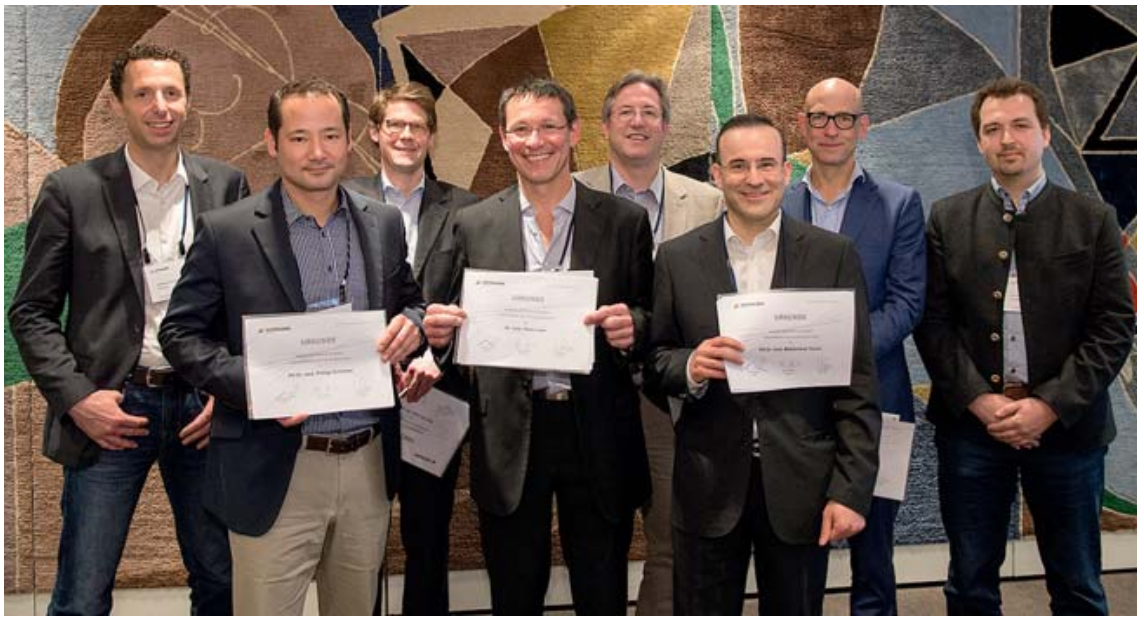

Abb. 8 Neu aufgenommene Akademiemitglieder (Foto: Jürgen Staiger).

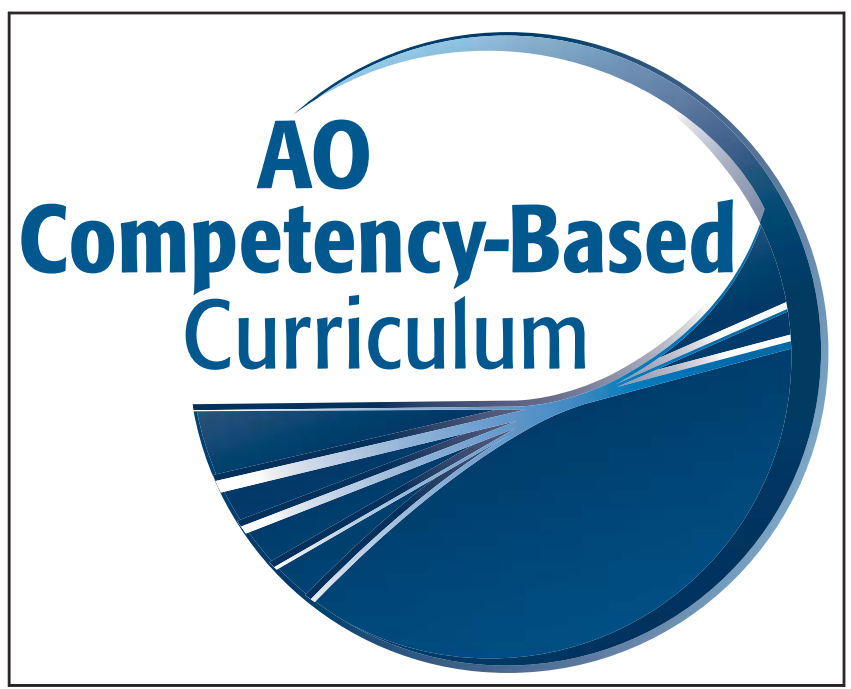

Abb. 9 Auszeichnung für Erfüllung der AO-Vorgaben.
Für die Schulung der AO Faculty gibt es ein Faculty Education Program (FEP) und für Kursleiter ein Chairmen Training Program (CTP). AOT Faculty an internationalen AO-Kursen, z.B. in Davos, müssen ein FEP absolviert haben, Chairmen ein CTP. In Deutschland werden 2 FEP Kurse pro Jahr angeboten. Zusätzlich gibt es individuelles Coaching für Faculty.

AOT Education hat internationale Mindestanforderungen der Curricula für die AOT-Basis-/-Prinzipien-Kurse sowie die AOT-Fortgeschrittenen-, -Hand- und -ORP-Kurse definiert (weitere werden folgen). Die von den Kursleitern eingereichten Programme werden von AOT Education International begutachtet und nur bei Erfüllung der Vorgaben gibt es die Auszeichnung „AO CompetencyBased Curriculum.“

Ein Disclosure Statement/Erklärung potenzieller Interessenskonflikte der Faculty wird im Laufe des Jahres eingeführt.
Das ORP Chapter war in 2015 sehr aktiv mit 7 Traumakursen und 2 ORP-Tagen. Auf der jährlichen ORP-Mitgliederversammlung wurden 6 neue Faculty-Mitglieder aufgenommen.

\section{Fellowships und Reisestipendium}

Aktuell gibt es 28 AOTrauma-FellowshipZentren in Deutschland (Vorjahr 24). Im Jahr 2015 kamen 65 Fellows nach Deutschland (2014: 66), davon 16 aus Europa (2014: 9), 23 aus Asien (2014: 34), 1 aus Lateinamerika (2014: 3), 6 aus Nordamerika (2014: 5) und 19 aus dem Nahen Osten (2014: 15). Damit waren 54\% aller AOTrauma Fellows, die nach Europa kamen, an einer deutschen Klinik.

Ausbaufähig ist die Anzahl der deutschen Ärzte, die sich für Fellowships im Ausland bewerben. In 2015 wurden 3 deutsche Fellows gefördert (2014: 2). Aktuell läuft die Ausschreibung für die
Fellowships 2017 (siehe separaten Artikel „Ausschreibungen“).

Die Reisestipendien 2016 der AOT Deutschland wurden im Rahmen der Jahrestagung vergeben an Baumann (Regensburg), Gehrmann (Düsseldorf), Oeckenpöhler (Münster) und Wagner (Mainz).

\section{Forschung und Wissenschaftspreis}

Im Jahr 2015 wurden 2 Calls durchgeführt. Aus 24 Anträgen wurden von der Auswahlkommission 6 Projekte ausgewählt und mit einem Gesamtvolumen von $75000 €$ gefördert.

Der Wissenschaftspreis 2015 wurde an Dr. Stefan Zwingenberger (Dresden) vergeben für seine Arbeit über „Enhancement of BMP-2 Induced Bone Regeneration by SDF-1a Mediated Stem Cell Recruitment“.

Für den diesjährigen Preis werden noch Bewerbungen bis zum 31. August entgegengenommen (siehe separaten Artikel „Ausschreibungen“).

\section{Neue Nachwuchsförderung zum 1. Mal verliehen}

Auf der Jahrestagung fand die Auswahlsitzung Forschungsförderung statt, auf der zum 1. Mal 2 Förderstipendien (max. $12000 €$ pro Person) vergeben wurden. Eingereicht werden durften sowohl klinische als auch experimentelle Forschungskonzepte aus den Bereichen Traumatologie, MKG und VET von jungen (bis zum Alter von 35 Jahren), klinisch aktiven Unfallchirurgen, die in Deutschland an Universitätskliniken, regionalen und kommunalen Krankenhäusern tätig sind.

Aus den 14 Bewerbungen wurden 3 Kandidaten zur Auswahlsitzung AO-Forschungsförderung nach Bern eingeladen. Jeder Kandidat hatte 6 Minuten, um die Arbeit vorzustellen. Danach erfolgte Diskussion und Auswahl. Prämiert wurden 1. Ferdinand Wagner (Freiburg, „Biomechanische Vergleichsanalyse von resorbierbaren Magnesiumschrauben mit Titanschraubenosteosynthese $\mathrm{zu}$ operativen Stabilisierung von Radiusköpfenfrakturen“) mit $10000 €$ und

2. Matthias Krause (Hamburg, „Expositionsmöglichkeiten des Tibiakopfes über verschiedene Zugänge am humanen Kadaverkniegelenk“) mit $12000 €$. 


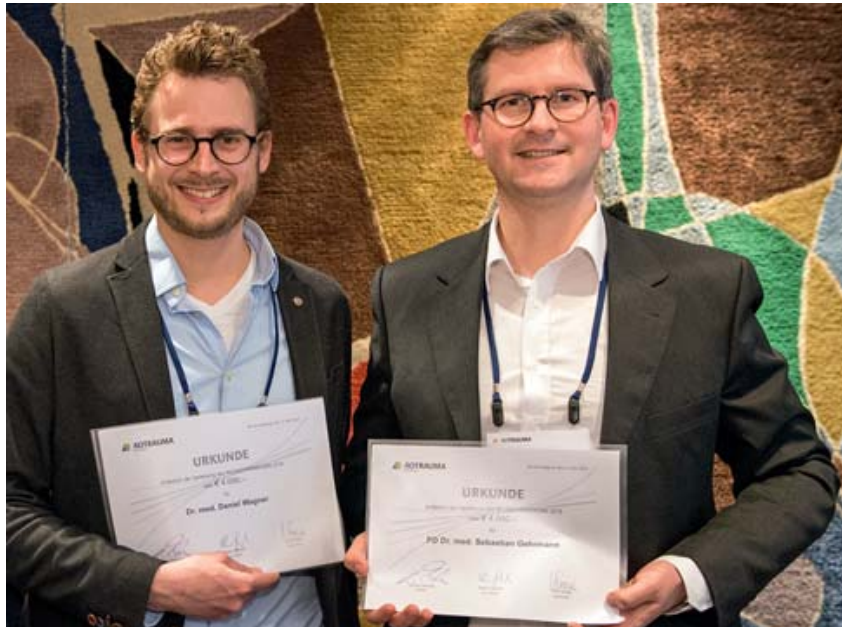

Abb. 10 Wagner und Gehrmann (Foto: Jürgen Staiger).

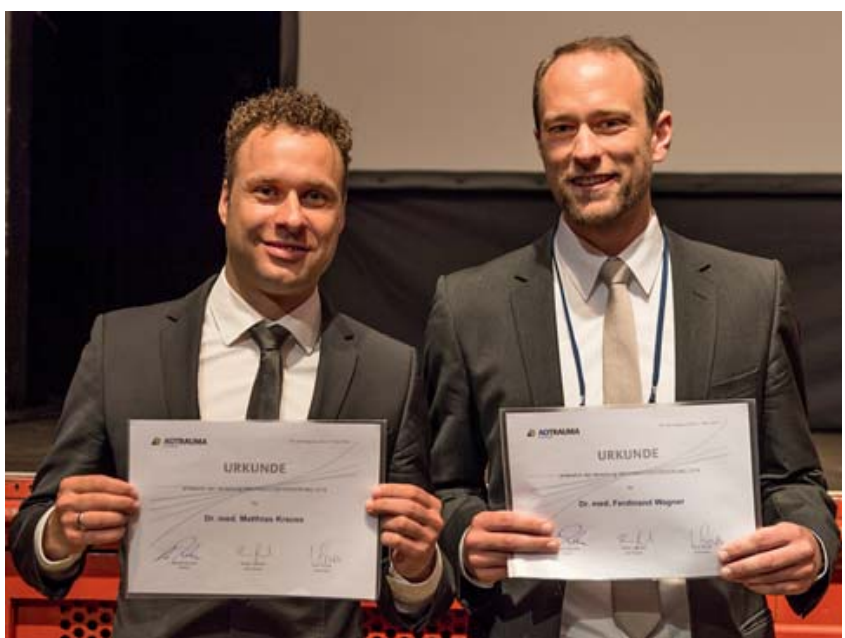

Abb. 12 Krause und Wagner (Foto: Jürgen Staiger).

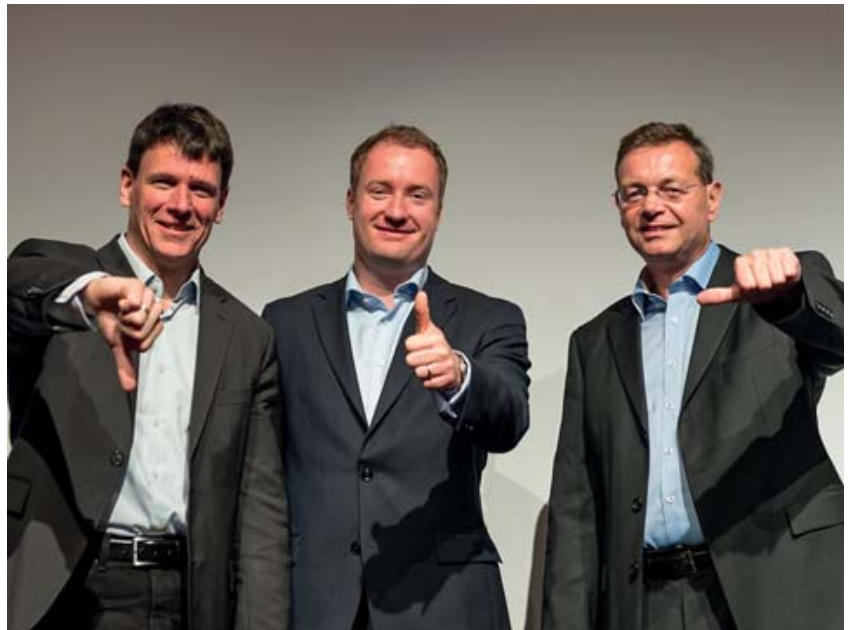

Abb. 11 Duda, Stange und Pape (Foto: Jürgen Staiger).

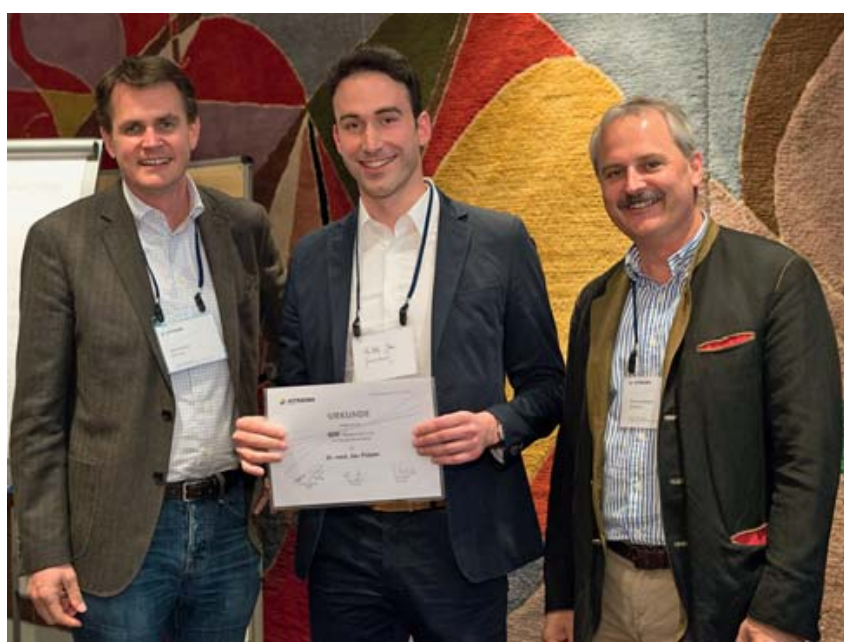

Abb. 13 Stöckle, Pützler und Gebhard (Foto: Jürgen Staiger).
Die 2. Ausschreibung in diesem Jahr endet am 31. August (siehe separaten Artikel „Ausschreibungen“).

\section{Mitglied}

Die AOTrauma Deutschland begrüßte auf der Jahrestagung ihr 600. Mitglied, Dr. Jan Pützler, Universitätsklinik Münster.

\section{Ausblick 2016}

Im laufenden Jahr werden sich die Aktivitäten der deutschen $\mathrm{AO}$ auf hohem Niveau stabilisieren. Die dynamische Entwicklung der letzten Jahre wird in nachhaltige Strukturen für die unterschiedlichen Zielgruppen konsolidiert. Dazu gehören in 2016 die ersten Aktivitäten der Akademie und in 2017 für die Zielgruppe Facharzt bis Leitender Oberarzt (ehem. Alumni). An der stetigen Verbesserung der - bereits schon sehr hohen - Qualität der Weiterbildungen wird durch Schulung der Faculty und Qualitätskontrolle bei den Kursinhalten gearbeitet.

Weitere Informationen zu allen Themen und Ausschreibungen sind erhältlich unter www.aotrauma.org/Deutschland
Prof. Dr. med. Michael J. Raschke

Direktor der Klinik für Unfall-, Handund Wiederherstellungschirurgie

Universitätsklinikum Münster (UKM) Albert-Schweitzer-Campus 1,

Gebäude W1

48149 Münster

Philip Wilbrandt

AOTrauma Deutschland

Langenbeck-Virchow-Haus Luisenstraße 58/59

10117 Berlin

p.wilbrandt@aodeutschland.de 